\title{
STUDIES ON THE ANTIGENIC PATTERNS OF BACTERIAL FLAGELLA
}

\author{
RINTARO NAKAYA, HISAO UCHIDA AND HIDEO FUKUMI
}

The National Institute of Health

(Received, July 8, 1952)

\section{INTRODUCTION}

It is well known that there are two kinds of Salmonella organisms in respect of flagellar antigens; the one is monophasic and the other is diphasic. The distinction is based upon whether organisms form colonies showing uniform behavior in respect of flagellar agglutinability or dissociating into two kinds of colonies different from each other. Further consideration reveals the existence of two apparently different antigenic patterns in flagella of Salmonella organisms, namely flagella with only one antigenic factor, for example S. paratyphi $A$ has only one flagellar antigen $a$ in its first phase, and the flagella, as the second type, with more than one antigenic factor, as for example $g, m$ in $S$. enteritidis or $e, n, x \ldots$ in $S$. abortus equi. In the latter case, the factors never dissociate into different colonial types and are always found in coexistance, therefore it is to be considered that each bacterial body is attached with the flagella containing every antigenic factor. Though it is evidenced that the H-antigen represents flagellar substances, there is no direct answer to the question as to whether the flagella of each bacterial body are uniform in respect of antigenic patterns, i. e. a single flagellum contains each of the antigenic factors altogether or there are several kinds of flagella differing from each other in respect of antigenic factors contained in them. The present investigation has been conducted to make this question clear utilizing purified flagellar suspension.

\section{EXPERIMENTAL METHODS}

The strains used: S. enteritidis 1891 and S. abortus equi beta 202.

The method of purification for flagella: The method previously described in the paper ${ }^{1)}$ was followed. 
The sera used: Anti- $(m, t)$ serum was prepared from H-serum of Salmonella oranienburg 9087 . Its agglutination titer was $1: 10,240$. Anti$(f, g)$ serum was prepared from H-serum of Salmonella derby. Its agglutination titer was 10,240. Anti- $(e, h)$ serum was prepared from antiserum of the first phase of $S$. reading. Its agglutination titer was $1: 51,200$.

Precipitation reaction of flagellar suspension with its antiserum: On half $\mathrm{ml}$ of flagellar suspension in phisiological saline was mixed with one half $\mathrm{ml}$ of antiserum and the result was read after it was kept standing overnight.

\section{EXPERIMENTAL RESULTS}

1. Experiments with purified flagella of Salmonella enteritidis 1891.

Table 1-a presents the precipitation reaction of various dilutions of purified flagellar suspension of Salmonella enteritidis with varying dilutions of anti- $(m, t)$ serum. The original suspension of purified flagella contained, in this case, $0.39 \mathrm{mg}$ per $\mathrm{ml}$ of nitrogen according to Kjeldahl method. In the Table, + means positive precipitation reaction and negative reaction, while $\oplus$ indicates that precipitation was complete; therefore the supernatant fluid was entirely clear. The above cyphers also apply to Tables 2 and 3. The Table indicates that the flagella with antigenic pattern $g, m$ were completely precipitated by anti- $(m)$ antibody in the anti- $(m, t)$ serum in the antibody-excess zone.

Table 1

Experiments with the flagella of Salmonella enteritidis, $(g, m)$

(a)

\begin{tabular}{|c|ccccccccc|}
\hline \multirow{8}{*}{$\begin{array}{c}\text { Serum } \\
(m, t) \\
\text { dilution }\end{array}$} & \multicolumn{7}{|c|}{ Antigen $(g, m)$} & dilution \\
\cline { 2 - 8 } & $2^{1}$ & $2^{2}$ & $2^{3}$ & $2^{4}$ & $2^{5}$ & $2^{6}$ & $2^{7}$ & $2^{8}$ & $2^{9}$ \\
\hline $5 \times 2^{0}$ & + & + & + & + & + & + & + & + & - \\
$5 \times 2^{1}$ & + & + & + & + & + & + & + & + & - \\
$5 \times 2^{2}$ & + & + & + & + & + & + & + & + & - \\
$5 \times 2^{3}$ & + & + & + & + & + & + & + & + & - \\
$5 \times 2^{4}$ & + & + & + & + & + & + & + & + & - \\
$5 \times 2^{5}$ & + & + & + & + & + & + & + & + & - \\
$5 \times 2^{6}$ & + & + & + & + & + & + & + & + & - \\
$5 \times 2^{2}$ & + & - & - & - & - & - & - & - & - \\
\hline
\end{tabular}


A similar experiment was performed with anti- $(f, g)$ serum and the same flagellar material. Table 1-(b) presents the result of this experiment, which also indicates that the flagella with antigenic pattern $g, m$ were entirely precipitated by the antibody $(g)$ in anti- $(f, g)$ serum in the antibody-excess zone.

Table 1

(b)

\begin{tabular}{|c|ccccccccc|}
\hline \multirow{8}{*}{$\begin{array}{c}\text { Serum } \\
(f, g) \\
\text { dilution }\end{array}$} & \multicolumn{8}{|c|}{ Antigen $(g, m)$ dilution } \\
\cline { 2 - 9 } & $2^{1}$ & $2^{2}$ & $2^{3}$ & $2^{4}$ & $2^{5}$ & $2^{6}$ & $2^{7}$ & $2^{8}$ & $2^{9}$ \\
\hline $5 \times 2^{0}$ & + & + & + & + & + & + & + & + & - \\
$5 \times 2^{1}$ & + & + & + & + & + & + & + & + & - \\
$5 \times 2^{2}$ & + & + & + & + & + & + & + & + & - \\
$5 \times 2^{3}$ & + & + & + & + & + & + & + & + & - \\
$5 \times 2^{4}$ & + & + & + & + & + & + & + & + & - \\
$5 \times 2^{5}$ & - & - & - & - & - & - & - & - & - \\
\hline
\end{tabular}

From the above mentioned experimental results, it is concluded that the flagellar material with antigenic pattern $g, m$ of $S$. enteritidis 1891 contains both $g$ and $m$ factors in a single flagellum together, and that a flagellum possessing only one single antigenic factor, either $g$ or $m$, never exists in the case of this organism.

2. Experiments with purified flagella of Salmonella abortus equi beta 202.

The result of precipitation reaction using varying amounts of purified flagellar suspension of Salmonella abortus equi beta 202 with varying dilution of anti- $(e, h)$ serum is presented in Table 2 . In this case, the original flagellar suspension contained $0.45 \mathrm{mg}$ per $\mathrm{ml}$ of nitrogen. From the results, it has become clear that the flagella with antigenic pattern $e, n, x$ are completely precipitated by the antibody $(e)$ in anti- $(e, h)$ serum in the antibody-excess zone. The above fact indicates that each flagellum of Salmonella abortus equi beta 202 contains all of the antigenic factors $e, n$ and $x$. In other words, the antigenic pattern $e, n, x$ is applicable to a single flagellum, and that there is no flagellum of this organism found possessing only one or two of these antigenic factors $e, n$ or $x$. 
Table 2

Experiment with the flagella of Salmonella abortusequi $(e, n, x \ldots)$

\begin{tabular}{|c|c|c|c|c|c|c|c|c|c|}
\hline \multirow{2}{*}{$\begin{array}{l}\text { Serum } \\
(e, h) \\
\text { dilution }\end{array}$} & \multicolumn{9}{|c|}{ Antigen $\left(e, n, x_{\ldots} ..\right)$ dilution } \\
\hline & $2^{1}$ & $2^{2}$ & $2^{3}$ & $2^{4}$ & $2^{5}$ & $2^{6}$ & $2^{7}$ & $2^{8}$ & $2^{9}$ \\
\hline $5 \times 2^{0}$ & + & + & + & + & + & + & + & + & - \\
\hline $5 \times 2^{1}$ & + & + & + & + & + & + & + & + & - \\
\hline $5 \times 2^{2}$ & - & - & - & - & - & - & - & - & - \\
\hline
\end{tabular}

Table 3

Experiment with the mixed flagella $((g, m)$ and $(e, n, x \ldots))$

\begin{tabular}{|c|c|c|c|c|}
\hline First Serum & $\begin{array}{c}\text { Antigen } \\
\left(\begin{array}{c}(g, m) 1: 16 \\
(e, n, x \ldots) 1: 8\end{array}\right)\end{array}$ & Supernatant & Second Serum & Supernatant \\
\hline$(m, t) 1: 20$ & + & crowdy & $(e, h) 1: 5$ & clear + \\
\hline$(e, h) 1: 5$ & + & crowdy & $(m, t) 1: 20$ & clear + \\
\hline
\end{tabular}

3. Experiments with a mixed suspension of the flagella from both S. enteritidis 1891 and $S$. abortus equi beta 202 .

As seen from the above mentioned experiments, the dilution factor of antiserum, at which precipitation reaction turns up positive with puriffed flagellar suspension, is pretty higher than in $\mathrm{H}$-agglutination reaction with bacterial suspension. Therefore a question arises as to whether the complete precipitation of flagella in antibody-excess zone in the above mentioned experiments was a purely specific antigen-antibody reaction or even those flagella containing no antigen corresponding to the antibodies in antiserum used were precipitated together with the flagella reacting with their corresponding antibodies in the serum by clumping effect. The following experiments were performed to give an answer to this question.

A purified flagellar suspension of Salmonella enteritidies 1891 (1:4 diluted from the original suspension with nitrogen content of $0.39 \mathrm{mg}$ per $\mathrm{ml}$ ) and that of Salmonella abortus equi beta 202 (1:2 diluted from the original suspension with nitrogen content of $0.45 \mathrm{mg}$ per $\mathrm{ml}$ ) were mixed in equal amount and $0.5 \mathrm{ml}$ of the mixture was added with $0.5 \mathrm{ml}$ of $1: 10$ diluted anti- $(m, t)$ serum. In this case, although precipitation 
occurred, the supernatant did not become completely clear, indicating that precipitation reaction was not complete. The reactant suspension was subjected to centrifugation at 3,000 R.P.M. and $0.5 \mathrm{ml}$ of the supernatant was mixed with $0.5 \mathrm{ml}$ of anti- $(e, h)$ serum at $1: 2.5$ dilution. Precipitation reaction occurred and the supernatant became entirely clear this time. It has become clear herefrom that turbidity in the supernatant after the first reaction was due to the flagella capable of reacting with anti- $(e, h)$ serum.

In case, the mixed flagellar suspension was added first with anti- $(e, h)$ serum, although precipitation reaction occurred, the supernatant was also not clear. This reactant was centrifuged and the supernatant was mixed with anti- $(g, m)$ serum. This time, precipitation reaction was complete and the supernatant became completely clear.

From these experiments, it was concluded that, when mixed suspension of flagella different from each other in respect of antigenic pattern was mixed with antiserum corresponding to only either one of these antigens possessed by the flagella used, the flagella possessing antigens other than those homologous to the antiserum were never precipitated together. Therefore it is clear that the precipitation reaction observed in the experiments 1) and 2) are considered to be entirely specific.

\section{DISCUSSION}

The question as to whether antigenic factors are located in each single flagella of a single bacterial body, and consequently each flagella of a single bacterial body has one and the same antigenic pattern or several kinds of flagella showing varying antigenic patterns were attached to a single bacterial body was subjected to serological analysis utilising purified flagellar suspension.

Our experimental results confirmed that the first assumption of those above mentioned is true. In other words, among the schematic diagrams of Fig. 1, the diagram (a) is showing the true picture. 

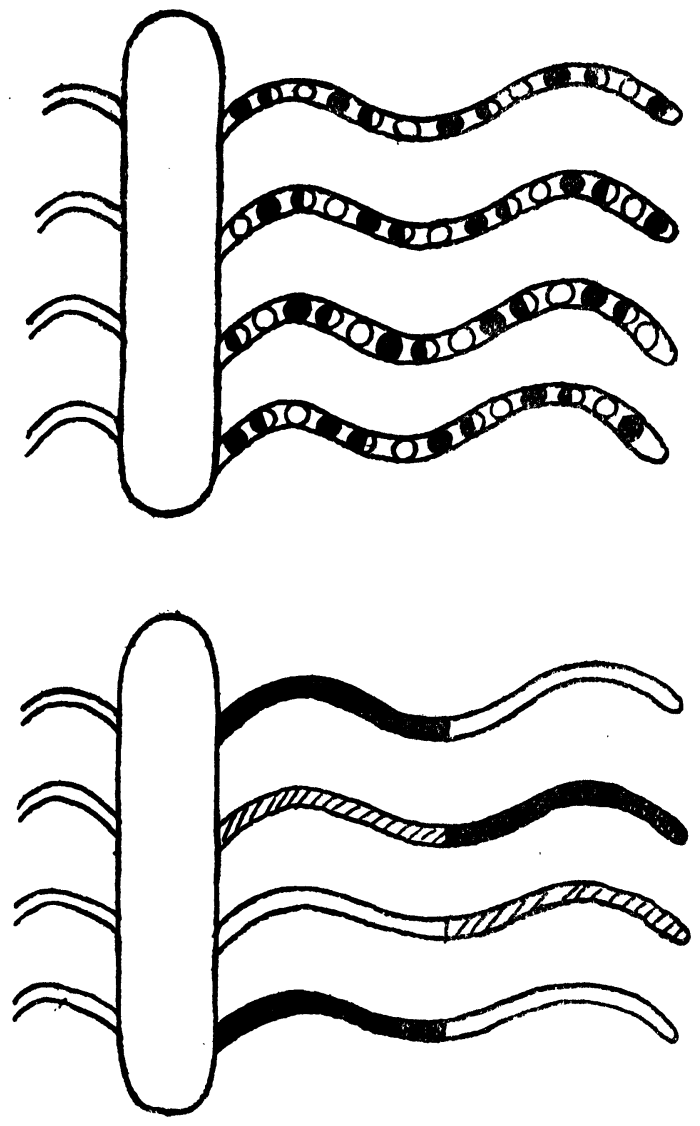

(b)

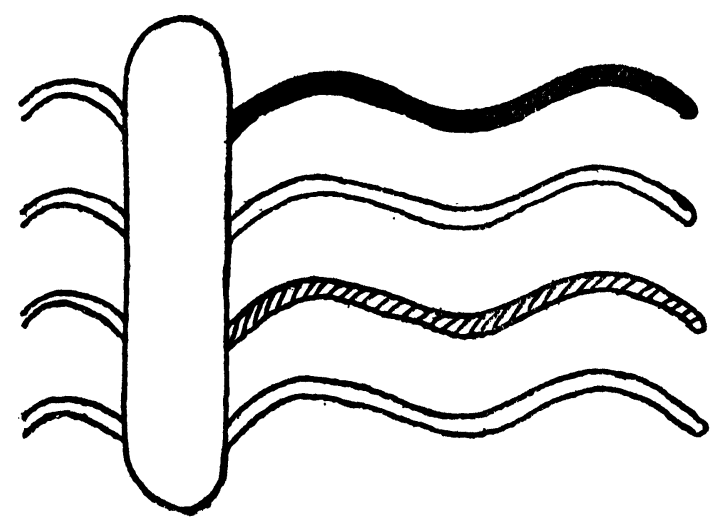

(c) 


\section{SUMMARY}

An analytical study of antigenic patterns of bacterial flagella was conducted using purified flagellar suspension of Salmonella organisms.

\section{REFERENCE}

Uchida, H. and Fukumi, H.: On the purification of bacterial flagella, Jap. J. med. Biol. Sei., in press. 